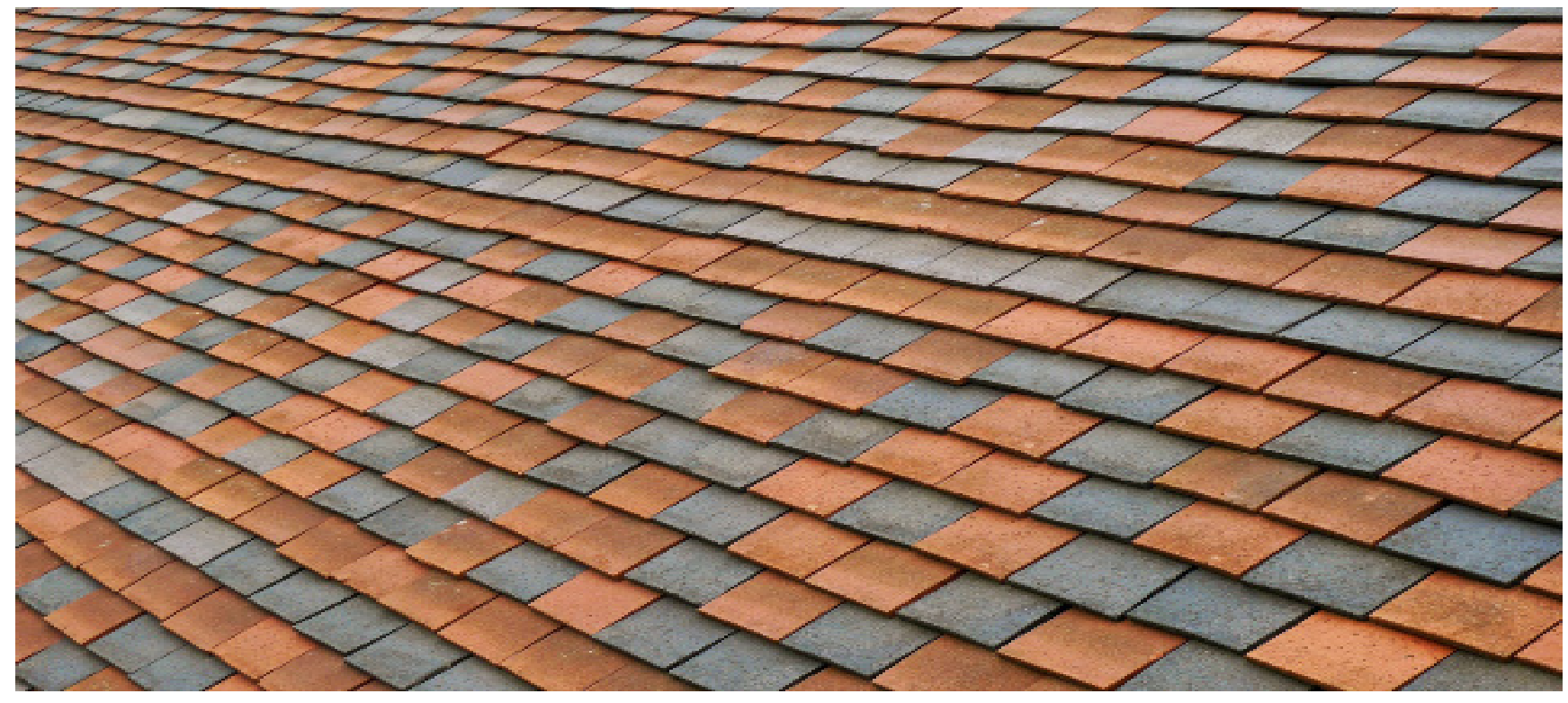

\title{
Diseño de teja modular ventilada en resina y arcilla sin cocer
}

\section{Ventilated modular tile design in resin and unfired clay}

\section{Natalia Estefanía Daza-Medina}

Programa de Arquitectura / Facultad de Ciencias, Artes y Humanidades, Semillero de Investigación EcoHabitat, nataliaestefaniadm@ufps.edu.co, https://orcid.org/0000-0003-0722-1204, Universidad Francisco de Paula Santander, Cúcuta - Colombia.

Angela María Montoya-lbarra

Programa de Arquitectura / Facultad de Ciencias, Artes y Humanidades, Semillero de Investigación EcoHabitat, angelamariami@ufps.edu.co, https://orcid.org/0000-0001-7519-3159, Universidad Francisco de Paula Santander, Cúcuta - Colombia.

Ender José Barrientos-Monsalve

Arquitecto, Doctor en Ciencias Gerenciales, Enderjosebamo@ufps.edu.co, https://orcid.org/0000-0001-6673-0223, Universidad Francisco de Paula Santander, Cúcuta - Colombia. 
Resumen

En muchas ocasiones se han buscado alternativas a los materiales con que se cubren las viviendas, y uno de los materiales más usados es la teja criolla, un material hecho con barro dándole forma y horneado después, es por ello que el objetivo de este trabajo fue proponer una teja en resina y arcilla sin cocimiento alguno, con esto se pretende mejorar el cómo se cubren las viviendas y ahorrando energía en su cocción, este trabajo fue de corte documental y descriptivo, ya que se analizaron materiales los cuales se propusieron para la realización de la teja, con esto se espera mejorar el desempeño de la teja antes utilizada con base a conseguir un material con un menor peso y menor costo.

Palabras clave: Arcilla, modulo, diseño, ventilación.
Abstract

On many occasions, alternatives to the materials used to cover the houses have been sought, and one of the most used materials is the creole tile, a material made with clay giving it shape and baked afterwards, that is why the objective of this work was to propose a tile in resin and clay without any baking, This work was documentary and descriptive, since the materials proposed for the production of the tile were analyzed. It is hoped that this will improve the performance of the tile previously used, based on obtaining a material with a lower weight and lower cost.

Keywords: Clay, module, design, ventilation. 
Introducción

A lo largo de los años la arquitectura ha procurado responder de manera asertiva a las condiciones a las que se enfrenta según su ubicación [1]. Por ello, uno de los grandes retos que afronta al estar en un clima cálido tropical es al de brindar un confort térmico al interior de un espacio, buscando disminuir el uso de los sistemas de enfriamiento artificial que generan afectaciones al medio ambiente [2]. En busca de generar este confort, la elección de los materiales a ser usados en la edificación es fundamental.

Materiales vivos como la tierra han evidenciado una gran cantidad de beneficios térmicos en relación con otros métodos constructivos [3], uno de estos está reflejado en la característica higrotérmica que poseen las edificaciones desarrolladas bajo sistemas constructivos de este tipo: muros en adobe, tapia, entre otros, los cuales son elementos vivos con la capacidad de autorregularse [4].

Dentro de la definición básica de espacio, la cubierta juega un rol prioritario, dado que es la que brinda cobijo de las condiciones climáticas externas y, por ende, es la que recibe el impacto directo de estas.

En Norte de Santander, la industria de la cerámica es uno de los sectores económicos de mayor desarrollo [5] y es esta la que se encarga de la producción y distribución de productos como bloques, tejas, tableta vitrificada, enchapes, entre otros. Sin embargo, la ausencia de nuevas propuestas en términos de materiales y tendencias la ha convertido en una industria cada vez más obsoleta, a diferencia del desarrollo en países como España donde institutos como Innovarcilla [6], ASCER [7], entre otros, se preocupan por brindar innovación en el mercado.

Para el objetivo de este trabajo se enfocó en diseñar un sistema constructivo a través de la reinterpretación de los tipos de cubierta de teja más comercializados, generando una tipología modular elaborada a partir de la mezcla de materiales naturales y sintéticos con el fin de dar respuesta a las condiciones climáticas de la ciudad de Cúcuta, Norte de Santander. Y los específicos :

- Identificación de las tipologías de teja usadas en la ciudad.

- Reconocer las alternativas en materiales según sus propiedades fisicoquímicas para ser combinados con la arcilla local.

- Diseñar el prototipo teniendo en cuenta los beneficios de este en relación a los que se comercializan en la Ciudad.

- Configurar el sistema constructivo del prototipo.

Materiales y métodos

En primera instancia se realiza una revisión sobre las tipologías de cubierta en tierra disponibles en el mercado local según el libro del clúster de la cerámica [5] y se evidenciaron 3 tipologías de tejas cerámicas dominantes.

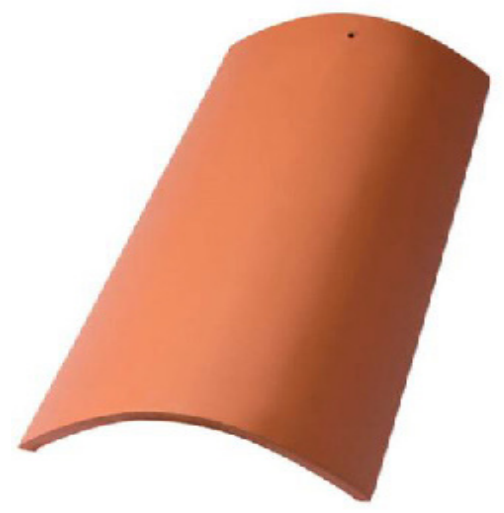

Figura 1: Teja Colonial - Distribuida por la Ladrillera Cúcuta

En la Figura 1 se encuentran las tejas coloniales (denominadas también árabes, alomadas o lomudsa), que son un tipo de tejas empleadas en la cobertura de cubiertas inclinadas. Son de forma troncocónica, obtenida al dar curvatura a un trapecio isósceles. Las dimensiones más 


\section{0}

corrientes son $45 \mathrm{~cm}$. de largo, por 21 y 16 de ancho y 8 de alto. Cada unidad posee un peso aproximado de $2 \mathrm{~kg}$ [8] [9]. En la Ciudad de Cúcuta, Norte de Santander, es producida y comercializada principalmente por la Ladrillera Cúcuta[5].

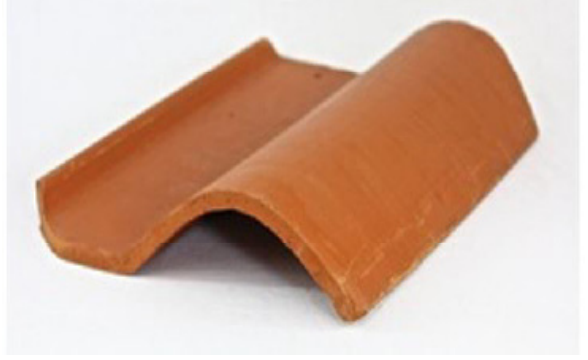

Figura 2: Teja Romana - Distribuida por Vitrificados de Cúcuta

Esta teja romana, en la figura 2, es usada para cubierta inclinada y cuenta con una parte central plana y los bordes laterales elevados para mejor sujeción[9], adicional a la sección curva con el mismo ángulo que la colonial.

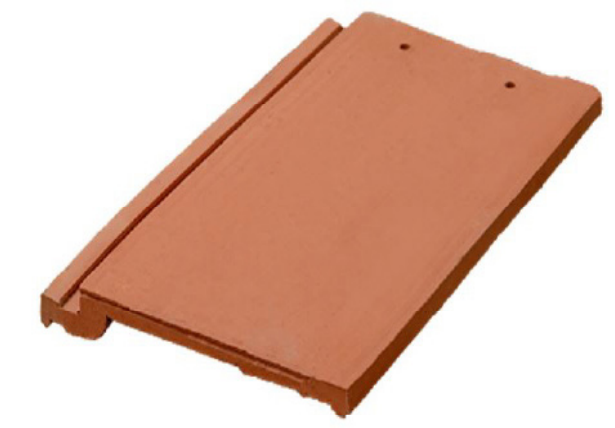

Figura 3: Teja Plana - Distribuida por el Tejar de Pescadero

Las tejas planas como la de la figura 3 son piezas conformadas por superficies lisas planas o con moldurados; su grosor es variable de acuerdo al modelo y material con el cual están hechas[9]. En el caso de las tejas cerámicas su instalación es a través de clavos que la fijan a la estructura, por lo que incluyen dos perforaciones para su respectiva instalación.

Sin embargo, a pesar de que la materia prima de estos elementos es la arcilla, estos son sometidos a un proceso de cocción que elimina una de las características principales de la tierra viva, como lo es su capacidad higrotérmica [4].

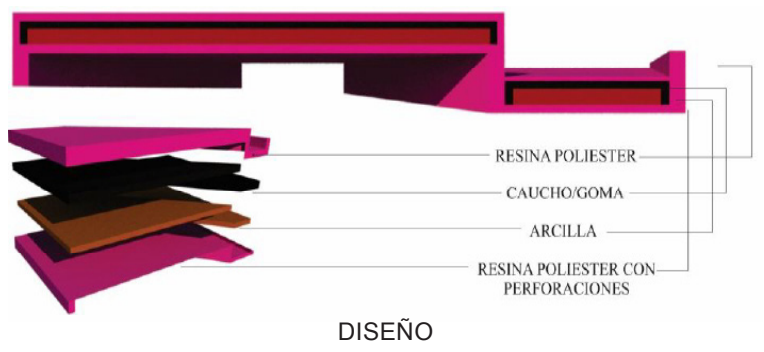

Figura 4: Capas de la Teja Ventilada - Fuente Propia

El elemento modular está conformado por una serie de capas como se aprecia en la figura 4 , cada una con un espesor dado según las propiedades del material de dicha capa; en la base esta la resina poliéster con perforaciones de $0.25 \mathrm{~mm}$ que permiten que la arcilla que esta sobre ella respire; la goma que aísla a la tierra de la temperatura que alcanza la resina en su reacción química con el catalizador al solidificarse, y finalmente cierra con una capa más de resina poliéster.

Los materiales que conforman el prototipo son:

Arcilla

La arcilla sin cocer es un material de múltiples beneficios que cuenta entre sus propiedades la adsorción/absorción, capacidad de intercambio catiónico, plasticidad y comportamiento térmico, entre otras [10]. Sin embargo, a lo largo de su implementación en el área de la construcción siempre ha tenido un gran contrincante: el agua y la humedad que esta representa.

Para aprovechar estas propiedades y solventar la debilidad del material ante el contacto con el agua, se procuró generar un vacío en el interior de la resina, que desarrolla el papel de material aislante a las condiciones exteriores, cuyo fin es ser ocupado con arcilla sin cocer, 
junto a las respectivas perforaciones que le permitan respirar.

\section{Resina poliester}

Entre sus características principales se encuentra la ligereza, resistencia a los rayos ultravioletas, al agua y a la corrosión. Según lo anterior, se plantea que la resina poliéster sea el recubrimiento del elemento modular que se encuentra en contacto directo con la intemperie, aislando el corazón de tierra y el interior de la construcción de las condiciones climáticas.

Dado que en el proceso de curado el material alcanza ciertas temperaturas debido al proceso exotérmico[11], se toma en cuenta la aplicación de un aislante interno adicional que proteja el corazón de tierra de la teja.

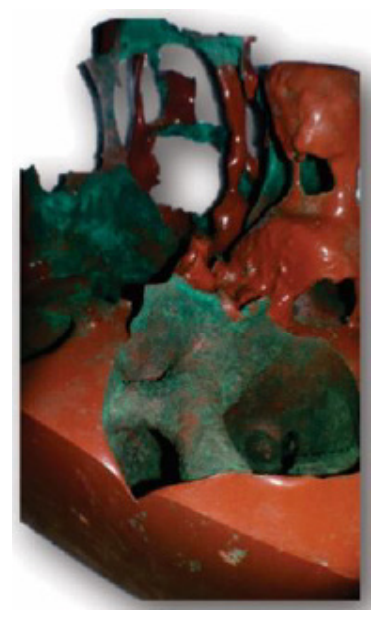

Figura 5: Experimentación con materiales - Resina Poliéster con chamota Fuente Propia

En aproximaciones anteriores al material (Figura 5), se realizaron pruebas de resistencia que demostraron que un elemento en resina poliéster con agregado de chamota, de $20 \mathrm{~cm}^{*} 20 \mathrm{~cm}^{*} 12 \mathrm{~cm}$ de dimensiones, con una relación de lleno y vacío de 70/30 resiste un peso de más de 80kg [11] , [12].
Resultados

Las dimensiones de la teja están dadas según la ergonomía de su manejo. En la figura 6 se aprecia que, con el fin de dar espacio a todas las capas internas que lo conforman, la altura total del módulo es de $19 \mathrm{~cm}$, por lo que se consideró un elemento cercano al cubo con unas dimensiones totales de $22 \mathrm{~cm}^{*} 24 \mathrm{~cm}$ en planta, y $19 \mathrm{~cm}$ de altura total. Las inclinaciones que permiten el paso del aire le brindan el aligeramiento necesario para restarle robustez. [13] [14].
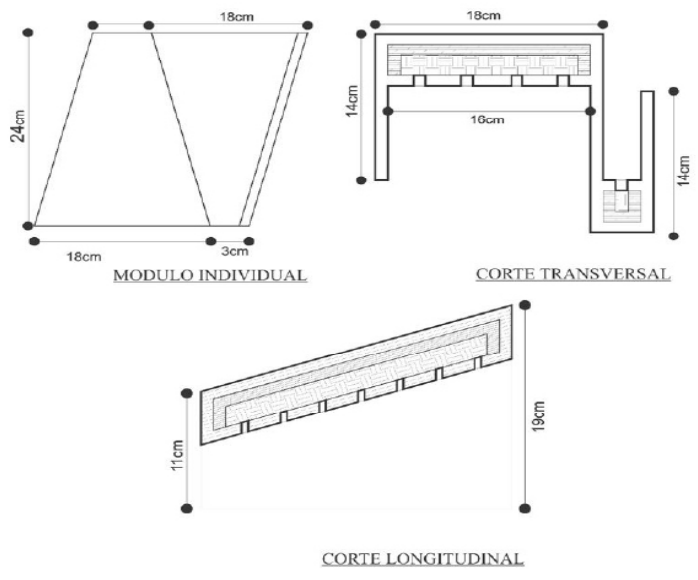

Figura 6: Dimensiones - Fuente Propia.

La teja modular es instalada sobre una base en machimbre, como se aprecia en la figura 7, y su disposición en instalación hace que se generen cámaras de aire que permiten la circulación de este a través de los vanos resultantes al apilar el módulo; esto resulta en una cubierta ventilada.

El método de instalación guarda una gran cantidad de similitudes con el que se usa para la instalación de la teja colonial, la diferencia principal es la rigurosidad que se debe manejar en lo que respecta a las distancias entre uno y otro elemento [15]. 


\section{2}

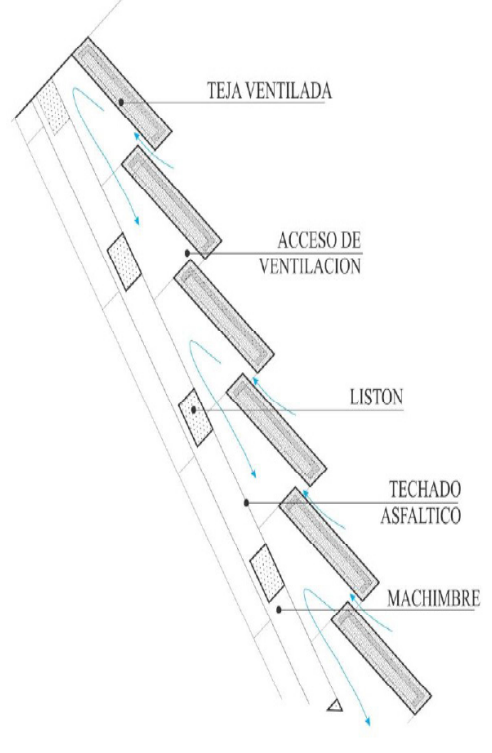

Figura 7: Detalle Constructivo - Fuente Propia.

El apilamiento (Figura 8) hacia los laterales está definido por una pestaña que genera un límite en el movimiento e instalación del mismo.

Su apariencia, como se evidencia en la figura 9 , es de una tonalidad terracota gracias a la mezcla de la Chamota con la resina poliéster y el catalizado

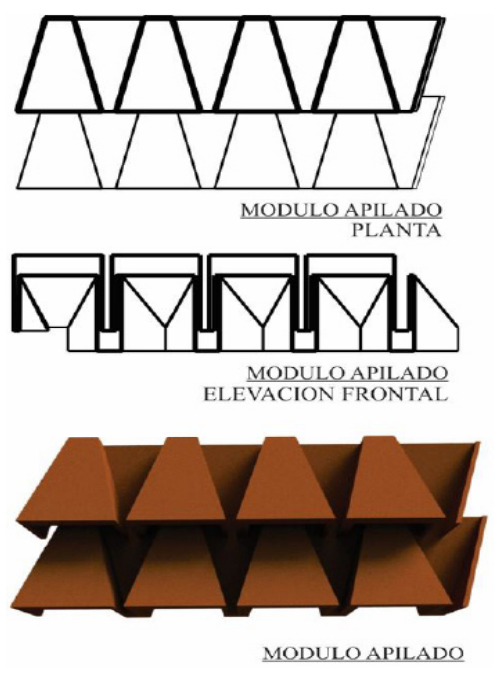

Figura 8: Especificaciones del Módulo Apilado - Fuente Propia.

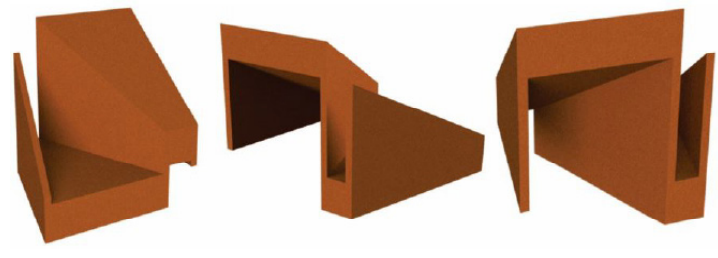

Figura 9: Modulo Individual - Fuente Propia

\section{Conclusiones}

Gracias a su resistencia a las condiciones climáticas del exterior y su durabilidad, la resina epoxy es un material ideal para ser aplicado en elementos de cubierta. Este por medio de su permeabilidad y dosificación permite ser flexible para el diseño de la forma que se propuso, tomando esto como consideración se pueden utilizar en muchos tipos de diseño arquitectónicos. Existen alternativas que le dan solución a las dificultades constructivas del uso de la tierra en las edificaciones contemporáneas; por ello se hace importante resaltar y priorizar los beneficios de esta.

\section{Referencias}

[1] B. Garzon, Arquitectura Bioclimatica. Buenos Aires: Nobuko, 2007

[2] A. Argemi, 7 razones para no usar el aire acondicionado. El Pais, 2013

[3] M. Diaz y M. Fernández, La certificación energética en edificaciones residenciales de tierra. Obra nueva y edificio existente. España, 2012

[4] L. Rivas Rodríguez, "Análisis del comportamiento higrotérmico de un muro de tapia mediante monitorización y simulación energética". Universidad da Coruña. Escola Universitaria de Arquitectura Técnica, España, 2015

[5] Cámara de Comercio, Documento del Clúster de Cúcuta, 2012 
[6] Innovarcilla. Centro tecnológico de la cerámica

[7] ASCER. Asociacion española de fabricantes de azulejos y pavimentos cerámicos

[8] Subgerencia Cultural del Banco de la República, Teja de barro, 2015

[9] HISPALYT. Catalogo de Soluciones Cerámicas.

[10] M. Pozo, Importancia de la composición y propiedades físicas de las arcillas en la preparación de peloides. IV CIBAP BOÍ, 2015

[11] A. Gil, Resinas de poliéster, 2015. [En línea]. Disponible en: http://www. eafit. edu. co/ servicios/centrodelaboratorios/infraestructura/ lab oratorios/Documents/Guia $\% \quad 20 \mathrm{de} \%$ 20manejo\% 20de \% 20resinas. pdf

[11] R.A. García, R. Bolivar León, y E.N. Florez Solano, "Validación de las propiedades físicomecánicas de bloques $\mathrm{H}-10$ fabricados en Ocaña Norte de Santander y la región", Revista

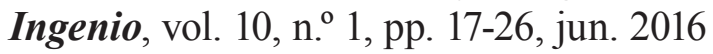

[12] R.A García-León, E.F. Solano, A. Acevedo, "Caracterización térmica de mezclas de arcillas utilizadas en la fabricación de productos de mampostería para la construcción”, Revista Tecnologías de Avanzada, vol.1, no. 31, pp. 2230,2018

[13] J. Sánchez-Molina, A. Sarabia-Guarin, y D.C. Alvarez-Rozo, "Evaluación de materias primas utilizadas en la fabricación de baldosas de gres en el sector cerámico de Norte de Santander (Colombia)", Respuestas, vol. 21, no. 2, pp. 48 -56 , jul. 2016

[14] V. Useche, G. Peña-Rodríguez, E. SeguraVenegas, y L. A. Gualdron-Sanchez, "Efecto de la concentración de sulfato de sodio en la viscosidad y difusividad térmica de barbotinas de arcillas rojas", Respuestas, vol. 14, n. ${ }^{\circ} 1$, pp. 50-57, may 2016
[15] J.F. Gelves-Diaz, J. Sánchez-Molina, y G. PeñaRodríguez, "Comportamiento de las arcillas del área metropolitana de Cúcuta sometidas a proceso de moldeo por extrusión", Respuestas, vol. 14 , n. 2 , pp. $32-38$, may 2016 